\title{
Decades Plantarum Novarum vel minus Cognitarum
}

by

\section{Gen-iti Koidzumi}

Prunus donarium Sieb. Ssp. speciosa Kordz. var praecox nov. var.

Floribus praecocioribus; foliis adultis minoribus; rami cortice rarius atro-purpurascente.

Nom. Jap. Tsukushi-Zakura.

Haв. Prov. Satsuma: Kajiki, in hortis cult.

Juncus prismatocarpus var. Leschenaultii Buch. svar. viviparus var. nov.

Fluitans vel submersus, capitulis viviparis.

Nom. JaP. Komotsi-Kogaizekisho.

Нав. Aomori ; Mido; Awa; Musashi ; Kiso ; Idsumi; Suwo; Hiuga; the Yayeyama-archipelago.

Vaccinium Smalli A. Gray, Bot. Jap. in Mem. Am. Acad. Art. Sci. VI, (1859) 398.

Foliis subtus ad costas medias primum laxe pubescentibus mox glabris, pilis incurvis vel subpatentibus; fructibus nigris.

Nom. Jap. Ohba-Sunoki.

Hab. Yezo; Saghalin; Nippon, Mt. Chokaisan.

var. glabrum nom. nov.

V. hirtum a typicum Maxim. Mel. Biol. VIII. 606.

Foliis subtus ab initio glabris, minoribus, plerumque ovatis.

Nom. Jap. Sunoki.

HAB. Japonia.

Vaccinium hirtum Thunb. Fl. Jap. (1784) 155.

V. Buergeri Mio. Ann. Mus. Bot. Lugd. Bat. I, 29. 
Folia subtus ad costas medias albo-pubescentia, pilis rectopatentibus. Fructus rubri vel demum nigri.

Nom. JaP. Usunoki.

HAB. Japonia.

var. lasiocarpum var. nov.

Folia subtus dense pubescentia. Fructus rubri pilosi.

Nom. Jap. Ke-Usunoki.

Hab. Nikko; Prov. Sanuki: Shozushima.

var. versicolor var. nov.

Folia supra intense viridia; fructibus primum rubris mox nigrescentibus.

Nom. JaP. Koba-Usunoki.

$\mathrm{H}_{\mathrm{AB}}$. Takahashimachi (Bittsiu); Tsurugisan (Awa); Ishidsutsiyama (Iyo).

var. atrum nov. var.

Flores pluri-racemosi; foliis subtus secus costas medias albo-tomentosis; fructibus nigris.

Nom. Jap. Kuromi-Usunoki.

HAB. Prov. Uzen : Higashi-okitamagori, Wadamura.

Spiraea chinensis Maxim. Act. Hort. Petrop. VI. (1.879) 1, p. 193.

S. Yatabei var. latifolia NAKAI in Bot. Mag. Tokyo XXIX. p. (228).

Nom. JaP. Toshimotsuke, Kibishimotsuke.

HAB. Chiugoku; Shikoku.

var. angustifolia (YATABE)

S. dasyantha var. angustifolia YATABE in Bot. Mag. Tokyo, VI. (1892) p. 348.

S. Yatabei NaKaI 1.c. (227.)

Nom. JaP. Hosoba-Ibukishimotsuke.

HaB. Shikoku: Prov. Awa, Nishiumura.

Brunella vulgaris $L$. var. albiflora nov. var.

Flores albi ; cet. ut in typo.

Nom. JAP. Shirobana-Utsubogusa.

Hab. Shikoku: Nishi-iyamura (Prov. Awa). 
Abelia spathulata $S$. et $Z$. var. tetrasepala nov. var.

Corolla ampla $3.5 \mathrm{~cm}$ longa; sepalis 4 late ellipticis vel late oblongis raro obovato-oblongis.

Nom. Jap. Oh-Tsukubane-utsugi.

Hab. Prov. Musashi, Chitsibu.

Cirsium riparium nom. nov.

C. Maximowiczii var. riparium Komz. in Bot. Mag. Tokyo, XXIX. 158.

Valde formosa; capitulis cernuis ; involucris intense laeteque viridibus, squamis intimis latis; floribus laete purpureoviolaceis; foliis incisis vel leviter pinnato-lobatis, inferioribus petiolatis, superioribus sessilibus non amplexicaulibus.

Caulis circ. 3-4-pedalis erectus, multistriatus, superne ramosus; ramis ornnibus erectis. Folia membranacea, ambitu oblonga, pinnatiloba lobis incisis, supra dense minuteque papillosa, subtus sparse araneosa, lamina ad petiolum longe desurrentia, margine spinulosa; foliis superioribus tantum pinnatilobis sessilibusque. Capitula magna $3.5-5.0 \mathrm{~cm}$. in diametro, ad apicem ramorum solitaria cernua; involucri squamis cire. 6-7 seriatis, extimis lanceolato-ovatis $7 \mathrm{~mm}$. longis, intimis lanceolato-linearibus $24 \mathrm{~mm}$. longis omnibus apice muticis; floribus purpureo-violaceis, corollae tubo limbum circ. dup superante. Antherae lineres exertae. Stigmata apice leviter bifida.

Nom. Jap. Sawa-Ohnoazami.

Hab. Prov. Uzen : Minami-okitamagori, Yatani; Prov. Iwashiro: ad pedem montis Adsumayama. Sendai.

Pyrus rufoferruginea nom. nov.

P. ferruginea KoInz. (non Hook. fil.) in Bot. Mag. Tokyo, XXIX. 158.

Nom. Jap. Mitsinoku-Nashi.

Haв. Hayatsinesan.

Spodiopogon sibiricus TRIN. var tomentosus nov. var.

Foliis subtus vaginisque adpresse villoso-tomentosis. 
Nom. JaP. Oh-Aburasusuki.

HAB. Prov. Hidaka : Samani.

Abelia (Zabelia, Biflorae) integrifolia sp. nov.

Frutex; ramis vetustioribus cinerascentibus; ramulis annotinis fuscis vel fusco-purpurascentibus; ramulis novellis pilosis. Folia membranacea, late vel anguste subrhombeo-oblanceolata, penninervia, sursum breviter cuneata, deorsum longe cuneatoattenuata, apice mucronata, lamina usque $4.5 \mathrm{~cm}$. longa $2.0 \mathrm{~cm}$. lata, margine integerrima et dense ciliata, subtus plerumque praecipue secus costas et venas dense pubescentia, supra pilosa; petiolis pubescentibus circ. 3-4 mm. longis, basi connatis. Pedunculi biflori in apice ramulorum solitarii, pilosi, $4-5 \mathrm{~mm}$. longi; pedicellis circ $2 \mathrm{~mm}$. longis pilosis; bracteolis brevissimis tripartitis, laciniis linearibus pilosis. Ovaria subteretia sparse pilosa. Sepala 4, lineari-lanceolata apice rotundata glabra, Corolla albida $1.3 \mathrm{~cm}$. longa campanulato-infundibuliformis, versus basin sensim angustata et sparse ciliata; limbo patulo rotundato roseo-maculato, circ. $6-7 \mathrm{~mm}$. diam.; stylis staminibusque inclusis. Achaenia subteretia, costata, $13 \mathrm{~mm}$. longa, glabra vel sparsissime pilosa.

Nom. Jap. Hosoba-Tsukubaneutsugi.

HAB. Prov. Bittsiu: Kawakamigori, Hongomura (VI. 22, 1915, Ipse!) ; Atetsugori, Kawanose (23, V. 1914, leg. Z. YoSHINo !).

DISTR. Species distinctissima, endemica!

Molinia japonica $\mathrm{H}_{\mathrm{ACK}}$. var. rupestris nov. var.

Humilis $15-20 \mathrm{~cm}$. alta; spiculae circ 8-15; lamina 2-3 mm. lata involuta, vaginis densius pubescentibus.

Nom. JaP. Ko-Numagaya.

Haв. Prov. Uzen : Mt. Adsumasan.

Salix (Fragiles) Matsudana sp. nov.

Haec species affinis S. fragili, sed ab ea differt foliis lanceolato-linearibus, ovariis sessilibus, stigmatis laciniis emarginatis.

Arbor? ramulis novellis sericeo-pubescentibus cito glabris; ramis vetustioribus glabris, cortice pallide fusco vel lutescente 
nitidoque vestitis. Folia vernalia linearia vel lineari-lanceolata, utrinque sub lente minute scabridula, supra glabriuscula, subtus adpresse sericea, apice acuminata, basi attenuata, margine integerrima vel remote mucronulato-serrulata; stipulis saltem in ramulis floriferis deficientibus; petiolis brevibus albo-sericeis. Amenta foliis coaetanea, breviter pedunculata, $2 \mathrm{~cm}$. longa; pedunculis pauci-foliatis rhachibusque albo-tomentellis; bracteolis ovatis apice obtusis vel obtusissimis extus infra medium albo-pubescentibus. Fl. $q$ : ovaria ovoideo-oblonga glabra, sessilia, stylis nullis; stigmatibus bilobis, laciniis leviter lobulatis; glandulis 2, anterioribus ovatis complanatis, posterioribus parvulis ovatis. Fl. $\hat{\delta}$ : stamina 2, filamentis basi albo-puberulis, glandulis 2 .

Hab. China: Kansu, Ranshiu (April 17, 1907, leg. Z. UmEMURA! no. 17).

Morus bombycis Koldz. nom. nov.

? M. japonica Sres. nom. nud! Syn. P1. Oec. Jap. (1827) p. 27, no. 163.

? M. japonica Nors. nom. nud! ex EndL. Gen. P1. Suppl. IV. pars 2, (1847) p. 33.

? M. japonica Aunib. nom. nud! ex Ser. Descr. et Cult. des Mûr. p. 226, (1855).

M. stylosa var. ovalifolia Ser. Descr. et Cult. des Mûr. (1855) p. 225, (Pro parte ex Bur.).

M. alba var. stylosa Bur. in DC. Prodr. XVII, (1873) p. 243.

Frutex vel arbor; foliis ovatis vel ovalibus basi cordatis, subito acuminatis dentato- vel duplicato-serratis, integris vel varie lobatis interdum dissectis, adultis supra scabro-asperatis, subtus secus nervos pubescentibus; stylis alte connatis ovarium circ. duplo superantibus, stigmate subulato puberulo stylo vix breviore vel subaequante. Inflorescentia $q$ pauci-pluriflora $3-\boldsymbol{\tau}$ $(-8) \mathrm{mm}$. longa ; $\hat{\delta}$ laxiflora $10-15 \mathrm{~mm}$. longa. Syncarpium atrum 5-13 $\mathrm{mm}$. longum, pedunculo aequelongo vel vix breviore.

Nom. JaP. Yamaguwa.

Distr. Japonica, Korea, China. 
Prunus nipponica Matstir. var. iwagiensis (Koenne)

P. iwagiensis Koenne Pl. Wils. II. (1912) 259.

Cupula turbinata.

HaB. Iwagisan, Hakkodasan.

var. pubescens nov. var.

Petiolis pubescentibus.

HaB. Iwatesan (=Ganjusan).

Prunus incisa Thunb. var. tomentosa nov. var.

Petiolis pedicellisque tomentosis.

Hab. Takaoyama (Musashi) ; circ. Yokohama.

var. serrata nov. var.

Foliis serratis longius acuminatis.

HAB. Hakoneyama, Takawoyama.

Salix Yoshinoi of sp. nov.

Quoad inflorescentia ad S. hirosakiensem affinis; sed foliis novellis subtus adpresse sericeo-villosis, adultis multinervis infra viridibus vel obscuriter glaucinis, pilis laxe persistentibusque.

Arbusculus, ramis annotinis brunneis, hornotinis cinerascentibus superne albo-tomentosis. Folia lanceolata breviter acuminata, basi rotundata rarius obtusa, multinervia, serrulata; juniora supra laxe subtus densissime villosa; adulta supra praeter costam mediam adpresse pilosam glabra, infra pilis plus minus persistentibus praecipue secus costas copiosioribus; lamina ad $9 \mathrm{~cm}$. longa $22 \mathrm{~mm}$. lata; petiolis circ. $5 \mathrm{~mm}$. longis albo-tomentosis; stipulis oblique ovatis acuminatis denticulatis. Inflorescentia foliis coaetanea $10-13 \mathrm{~mm}$. longa, pedunculis albo-tomentosis basi foliatis $5-7 \mathrm{~mm}$. longis; bracteolis ovatis apice rotundatis extus laxius puberulis. Fl. $q$ : ovario ovoideo albo-tomentoso sessile, stylis elongatis glabris, stigmatibus revolutis; glandula unica ovoidea.

Nom. JaP. Yoshino-Yanagi.

HAB. Prov. Bittsiu. Kawakamigori, Abe.

Polygonum japonicum Meiss. var. glandulosum nov. var.

Fertilis (sec. Yoshino); perianthium $3-4 \mathrm{~mm}$. longum album clandulosum, stylis longe exertis. 
Nom. Jap. Kibi-Sakuratade.

Hab. Prov. Bittsiu : Kibigori, Sosha.

Saussurea (Lagurostemon, Corymbiferae) imperialis sp. nov.

Rhizoma lignosum ascendens. Humilis perennans, caule 9$11 \mathrm{~cm}$. alto, crasso, simplice, striato, superne in corymbum confertum abeunte, a basi ad summum folioso, laxe araneosovilloso. Folia membranacea primum utrinque laxe araneosovillosa, mox subtus glabra, supra versus marginem parce puberula, margine inaequaliter aristato- vel mucronato-dentata ciliolataque, dentibus rarius falcatis, ovata vel lanceolato-ovata acuta vel subacuminata, basi late cuneata usque rotundata, per totum petiolum cuneatodecurrentia; radicalia longe (3-4 cm.), media breviter $(2-2.5 \mathrm{~cm}$.) superiora brevissime (circ. $5 \mathrm{~mm}$.) petiolata, summa sessilia, lamina 5-11 cm. longa $2.5-6$ cm. lata. Inflorescentia oligocephala, foliis lanceolato-linearibus suffulta. Capitula fere 10, campanulata, inter se arcte conferta, breviter (5 raro $25 \mathrm{~mm}$.) pedunculata, 10-14 $\mathrm{mm}$. longa, fere $8-15 \mathrm{~mm}$. diametro ; involucri squamis subquadriseriatis, atropurpureo-marginatis, superne breviter villosis, extimis ovatis cuspidatis, mediis late ovatis acutissimis, intimis lanceolatoovatis acutis. Flores videtur violacei, corolla $11 \mathrm{~mm}$. longa, tubo limbo aequilongo, limbo ad quatuor partes 5-fido, segmentis linearibus acutis; pappi serie externa subnumerosa ad $4 \mathrm{~mm}$. serie interna ad $9 \mathrm{~mm}$. longa. Achenium (immaturum) $3 \mathrm{~mm}$. longum nigro-fuscum laeve. Antherae basi sagitatae, caudis fasciculato-setosis.

Nom. JAP. Takane-kita-azami.

HaB. Yezo in alpibus Tokatsi-dake.

S. Riederi affinis, sed ab ea foliorum forma, anthoidio majore, squamae forma, caule humileque differt.

Cardamine Fauriei Fr. f. geifolia KoIdz.

C. geifolia KoIDz. Ic. P1. Kois. II. t. 97, (1914).

Нав. Yezo. 